\title{
Cooperative Fuzzy Games with a Coalition Structure and Interval Payoffs
}

\author{
Fanyong Meng* \\ School of Management, Qingdao Technological University, \\ Qingdao, 266520, Shandong, China \\ Qiang Zhang \\ School of Management and Economics, Beijing Institute of Technology, \\ Beijing, 100081, China \\ Yan Wang \\ Business School, Central South University, \\ Changsha, 410083, Hunan, China \\ Received 5 August 2011; accepted 3 January 2013
}

\begin{abstract}
Based on the extension Hukuhara difference between interval numbers, a generalized form of cooperative fuzzy games with a coalition structure and interval payoffs is proposed, which can be seen as an extension of crisp case. The interval Owen value for this kind of fuzzy games is studied, and its explicit form is given. When the fuzzy games are convex, the proposed interval Owen value is an interval population monotonic allocation function (IPMAF), and belongs to the associated core. Furthermore, we discuss a special kind of fuzzy games with a coalition structure and interval payoffs, and study the interval Owen value and the core of it. Some properties are also examined, which are coincided with the crisp case.
\end{abstract}

Keywords: Cooperative fuzzy game, Coalition structure, Interval Owen value, Extension Hukuhara difference

\section{Introduction}

The Shapley value (see [1]) is a well-known solution concept in cooperative game theory. Some researchers (see [2-6]) who have investigated the Shapley value have focused their attention on fuzzy coalition and realvalued characteristic function.

In some situations, players can only know imprecise information regarding the real outcome of cooperation. For this kind of games, Mares [7] and Vlach [8] researched games with crisp coalitions and fuzzy payoffs. Borkotokey [9] and Yu and Zhang [10] studied games with fuzzy coalitions and fuzzy payoffs. Since interval numbers can conveniently describe the lower and upper bounds of the coalition values. Alparslan Gök et al. [11, 12] and Branzei et al. [13] researched games with crisp coalitions and interval payoffs. Due to the limitation of fuzzy set operations, the discussions in [10-12] are all based on the Hukuhara difference (see [14]) for fuzzy sets directly or indirectly.

In 1977, Owen [15] introduced games with a priori unions which are known as the Owen coalition structures where the probability of cooperation among coalitions is considered. By extending the Shapley value for traditional games, Owen [15] proposed the Owen value for games with a coalition structure. The axiomatic systems of the Owen value are considered in [16-19]. Later, Owen [20] gave another payoff index for games with a coalition structure, which is called the Banzhaf-Owen value, and discussed its axiomatic system. More researches about axiomatic characterizations of the Banzhaf-Owen value can be

*Corresponding Author: mengfanyongtjie@163.com

This work was supported by the Natural Science Foundation Youth Project of China (No. 71201089), the National Natural Science Foundation of China (Nos. 71071018 and 71271217), the Natural Science Foundationl Youth Project of Shandong Province, China (ZR2012GQ005), and the Specialized Research Fund for the Doctoral Program of Higher Education (No. 20111101110036). 
seen in [21, 22]. Alonso-Meijide and Fiestras-Janeiro [23] pointed the Banzhaf-Owen value dissatisfies symmetry in quotient games, and gave another solution concept for games with a coalition structure, which is known as the symmetric Banzhaf value. But all above researches are only introduced for crisp case.

In this paper, based on the extension Hukuhara difference between interval numbers, we will research cooperative fuzzy games with a coalition structure and interval payoffs. As we will see, the extension Hukuhara difference between interval numbers can be used to more situations than the Hukuhara difference. Inspired by Sprumont [24], we show the given interval Owen value is an IPMAF when the fuzzy games are convex.

This paper is organized as follows: In the next section, we recall some notations and basic definitions, which will be used in the following. In section 3, an axiomatic definition of the interval Owen value for fuzzy games with a coalition structure and interval payoffs is proposed, and the explicit form of the interval Owen value is given. Meantime, some properties are researched. In section 4, we pay an attention to discuss the interval Owen value for a special kind of fuzzy games with a coalition structure and interval payoffs, which can be seen as an extension of fuzzy games introduced by Tsurumi et al. [4].

\section{Preliminaries}

\subsection{The Extension Hukuhara Difference between Interval Numbers}

Let $\mathbb{R}$ and $\mathbb{R}_{+}$be the sets of real numbers and positive real numbers, respectively.

Definition 2.1 Let $\bar{a}=\left[a^{-}, a^{+}\right]$, if $a^{-} \leq a^{+}$and $a^{-}, a^{+} \in \mathbb{R}$, then $\bar{a}$ is said to be an interval number; if $a^{-} \leq a^{+}$and $a^{-}, a^{+} \in \mathbb{R}_{+}$, then $\bar{a}$ is said to be a positive interval number.

Let $\bar{a}=\left[a^{-}, a^{+}\right]$and $\bar{b}=\left[b^{-}, b^{+}\right]$be two interval numbers. In order to ensure that $\bar{a}-\bar{b}$ is an interval number, the difference between $\bar{a}$ and $\bar{b}$ is defined as $\bar{a}-\bar{b}=\left[a^{-}-b^{+}, a^{+}-b^{-}\right]$.

In generally, we can not have $\bar{a}-\bar{b}+\bar{b}=\bar{a}$. The Hukuhara difference for fuzzy sets, proposed by Banks and Jacobs [14], can well deal with this problem. When it is applied to interval numbers, the Hukuhara difference is defined by:
Definition 2.2 Let $\bar{a}$ and $\bar{b}$ be two interval numbers, if there exists an interval number $\bar{c}$ such that $\bar{a}=\bar{b}+\bar{c}$, then $\bar{c}$ is called the Hukuhara difference between $\bar{a}$ and $\bar{b}$, denoted by $\bar{a}-_{H} \bar{b}=\left[a^{-}-b^{-}, a^{+}-b^{+}\right]$.

For example, let $\bar{a}=[2,5]$ and $\bar{b}=[4,6]$, since there exists the interval number $\bar{c}=[-2,-1]$ such that $[2,5]=$ $[4,6]+[-2,-1]$. We get $\bar{a}-{ }_{H} \bar{b}=[-2,-1]$, which is obviously different to $\bar{a}-\bar{b}=[-4,1]$.

Definition 2.3 Let $\bar{a}=\left[a^{-}, a^{+}\right]$and $\bar{b}=\left[b^{-}, b^{+}\right]$be two interval numbers, $\bar{a}$ is said to be an "imaginary" interval number if $a^{-}>a^{+}$, and $\bar{a}-_{H} \bar{b}$ is said to the "imaginary" Hukuhara difference between $\bar{a}$ and $\bar{b}$ if $a^{-}-b^{-}>a^{+}-b^{+}$.

For example, $\bar{a}=[5,3]$ is an "imaginary" interval number. Let $\bar{a}=[2,5]$ and $\bar{b}=[4,8]$. Since there does not exist an interval number $\bar{c}$ such that $\bar{a}=\bar{b}+\bar{c}$, we can not use the Hukuhara difference. If we do not consider the condition given in Definition 2.2, and adopt the Hukuhara difference between $\bar{a}$ and $\bar{b}$. Then we get $\bar{a}-_{H} \bar{b}=[-2,-3]$, which is a so-called "imaginary" interval number. And the Hukuhara difference between $\bar{a}$ and $\bar{b}$ is said to the "imaginary" Hukuhara difference

Definition 2.4 Let $\bar{a}=\left[a^{-}, a^{+}\right]$and $\bar{b}=\left[b^{-}, b^{+}\right]$be two interval numbers, then $\bar{a}-{ }_{e H} \bar{b}$ is called the extension Hukuhara difference between $\bar{a}$ and $\bar{b}$, where $\bar{a}-_{e H} \bar{b}=$ $\left[a^{-}-b^{-}, a^{+}-b^{+}\right]$.

From Definition 2.4, we know when there exists an interval number $\bar{c}$ such that $\bar{a}=\bar{b}+\bar{c}$, then their extension Hukuhara difference is equal to their Hukuhara difference. Otherwise, the extension Hukuhara difference between $\bar{a}$ and $\bar{b}$ is equal to their "imaginary" Hukuhara difference.

For convenience, in this paper, the sets of positive interval numbers and positive imaginary interval numbers are denoted by $\overline{\mathbb{R}}_{+}$. Moreover, the difference between interval numbers adopts the extension Hukuhara difference. If there is no fear of confliction, we still use $\bar{a}-{ }_{H} \bar{b}$ to denote the extension Hukuhara difference between the interval numbers in $\overline{\mathbb{R}}_{+}$.

Definition 2.5 Let $\bar{a}$ and $\bar{b}$ be two interval numbers, we write 
(1) $\bar{a} \geq \bar{b}$ if and only if $a^{-} \geq b^{-}$and $a^{+} \geq b^{+}$;

(2) $\bar{a}=\bar{b}$ if and only if $a^{-}=b^{-}$and $a^{+}=b^{+}$.

\subsection{Some Basic Concepts on Crisp Games with a Coalition Structure}

Let $N=\{1,2, \ldots, n\}$ be the set of players. The crisp coalitions in $N$ are denoted by $S_{0}, \ldots$, where $S_{0}=\left\{i \mid i \in S_{0}\right.$ s.t. $i \in N\}$, i.e., $S_{0}$ is formed by the players belong to it. The power set of all crisp subsets in $N$ is denoted by $P(N)$. For any $S_{0} \in P(N)$, the cardinality of $S_{0}$ is denoted by low case $s$. A function $v_{0}: P(N) \rightarrow \mathbb{R}_{+}$, satisfying $v_{0}(\varnothing)=0$, is called a set function. By $G_{0}(N)$, we denote the set of all set functions in $N$.

A crisp coalition structure $\Pi=\left\{B_{0}^{1}, B_{0}^{2}, \ldots, B_{0}^{m}\right\}$ in $N$ is a partition of $N$, i.e., $\cup_{1 \leq j \leq m} B_{0}^{j}=N$ and $B_{0}^{i} \cap B_{0}^{j}=\varnothing$ for all $i, j \in M=\{1,2, \ldots, m\}$ with $i \neq j$. A crisp coalition structure in $N$ is denoted by $(N, \Pi)$. For any $S_{0} \in$ $P(N, \Pi), S_{0}$ is called a feasible coalition, where $P(N, \Pi)$ denotes the set of all feasible coalitions in $(N, \Pi)$.

Owen [15] gave the Owen value for crisp games with a coalition structure as follows:

$$
\begin{gathered}
\beta_{i}\left(N, v_{0}, \Pi\right)=\sum_{R \subseteq M \backslash k} \sum_{S_{0} \subseteq B_{0}^{k} \backslash i} \alpha_{m}^{r} \beta_{b_{k}}^{s}\left(v_{0}\left(S_{0} \cup Q_{0} \cup i\right)\right. \\
\left.-v_{0}\left(S_{0} \cup Q_{0}\right)\right) \quad \forall i \in N,
\end{gathered}
$$

where $\alpha_{m}^{r}=\frac{r !(m-r-1) !}{m !}, \beta_{b_{k}}^{s}=\frac{s !\left(b_{k}-s-1\right) !}{b_{k} !}, m$ and $r$ denote the cardinalities of $M$ and $R$, respectively, $Q_{0}=\cup_{p \in R} B_{0}^{p}$ and $\Pi=\left\{B_{0}^{1}, B_{0}^{2}, \ldots, B_{0}^{m}\right\}$.

\subsection{Some Basic Concepts for Fuzzy Games with a Coalition Structure and Interval Payoffs}

The set of all fuzzy coalitions in $N$ is denoted by $L(N)$. The fuzzy coalitions in $L(N)$ are denoted by $S, \ldots$, i.g., $S=\{S(i) \mid i \in N\}$, where $S(i)$ indicates the membership grade of $i$ in $S$, i.e., the rate of the $i$ th player in $S$. For any $S \in L(N)$, the support is denoted by SuppS $=\{i \in N \mid$ $S(i)>0\}$, and the cardinality is denoted by $|\operatorname{Supp} S|$. We use the notation $S \subseteq T$ if and only if $S(i)=T(i)$ or $S(i)=$ 0 for all $i \in N$. For all $S, T \in L(N), S \vee T$ denotes the union of fuzzy coalitions $S$ and $T$, namely, $i \in$ $\operatorname{Supp}(S \vee T)$ if and only if $i \in \operatorname{Supp} S \cup \operatorname{Supp} T$ and $(S \vee T)(i)=S(i) \vee T(i) ; S \wedge T$ denotes the intersection of fuzzy coalitions $S$ and $T$, namely, $i \in \operatorname{Supp}(S \wedge T)$ if and only if $i \in \operatorname{Supp} S \cap \operatorname{Supp} T$ and $(S \wedge T)(i)=S(i) \wedge T(i)$.

In the following, we will use $S=\left\{S\left(i_{1}\right), S\left(i_{2}\right), \ldots, S\left(i_{n}\right)\right\}$ to denote the fuzzy coalition $S \in L(N)$. Furthermore, the braces will be omitted for singletons, e.g. by writing $S$,
$S \vee(\wedge) T, U(i)$ instead of $\{S\},\{S\} \vee(\wedge)\{T\},\{U(i)\}$ for any $\{S\},\{T\},\{U(i)\} \in L(N)$, where $U$ is a fuzzy coalition in $N$.

Similar to the definition of the coalition structure for crisp case introduced by Owen [15], we give the definition of the coalition structure for fuzzy coalitions as follows:

Definition 2.6 Let $U \in L(N), \Gamma$ is said to be a coalition structure on $U$, if $\Gamma$ satisfies

(1) $\vee_{k=1}^{m} B_{k}=U$,

(2) $\operatorname{Supp}_{P} \cap \operatorname{Supp}_{q}=\varnothing \quad \forall p, q \in\{1,2, \ldots, m\}$ s.t. $p \neq q$,

where $\Gamma=\left\{B_{1}, B_{2}, \ldots, B_{m}\right\}$ and $1 \leq m \leq|\operatorname{Supp} U|$, denoted by $(U, \Gamma)$.

For any $(U, \Gamma)$, the set of the feasible coalitions in $(U, \Gamma)$ is denoted by $L(U, \Gamma)$.

Example 2.1 Let $N=\{1,2,3,4\}$, and $U=\{U(i)\}_{i \in N}$ be a fuzzy coalition in $L(N)$ such that $U(i)>0$ for any $i \in N$. $\Gamma=\left\{B_{1}, B_{2}\right\}$ is a fuzzy coalition structure in $U$, where $B_{1}=\{U(1), U(2)\}$ and $B_{2}=\{U(3), U(4)\}$, then $L(U, \Gamma)=\{\varnothing,\{U(i)\}(i \in N),\{U(1), U(2)\},\{U(3), U(4)\}$, $\{U(1), U(2), U(3)\},\{U(1), U(2), U(4)\},\{U(1)$, $U(3), U(4)\},\{U(2), U(3), U(4)\}, U\}$.

A function $\bar{v}: L(U, \Gamma) \rightarrow \overline{\mathbb{R}}_{+}$, satisfying $\bar{v}(\varnothing)=[0,0]$, is called an interval set function. All interval set functions on $L(U, \Gamma)$ are denoted by $I G(U, \Gamma)$.

Example 2.2 In Example 2.1, if $U(1)=0.3, U(2)=0.8$, $U(3)=0.5$ and $U(4)=0.6$, and the interval set function $\bar{v}$ is defined as follows:

$\bar{v}(\varnothing)=[0,0], \bar{v}(U(1))=[2,3], \bar{v}(U(2))=[1,2], \bar{v}(U(3))=$ $[2,4], \bar{v}(U(4))=[3,6], \bar{v}(U(1), U(2))=[5,7], \bar{v}(U(3), U(4))$ $=[9,12], \bar{v}(U(1), U(2), U(3))=[10,13], \bar{v}(U(1), U(2)$, $U(4))=[12,15], \bar{v}(U(1), U(3), U(4))=[13,15], \bar{v}(U)=[20$, 22].

Then it is an interval set function belongs to $I G(U, \Gamma)$.

Definition 2.7 Let $\bar{v} \in I G(U, \Gamma)$, if $\bar{v}^{B}\left(R_{0}\right)=\bar{v}\left(\vee_{r \in R_{0}} B_{r}\right)$ for any $R_{0} \subseteq M_{0}$, then $\bar{v}^{B}$ is said to be an interval quotient game in $(U, \Gamma)$, where $\Gamma=\left\{B_{1}, B_{2}, \ldots, B_{m}\right\}$ and $M_{0}=\{1,2, \ldots, m\}$, denoted by $\left(M_{0}, \bar{v}^{B}\right)$.

Definition 2.8 Let $\bar{v} \in I G(U, \Gamma)$ and $T \in L(U, \Gamma)$, if we 
have $\bar{v}(T \wedge S)=\bar{v}(S)$ for any $S \in L(U, \Gamma)$, then $T$ is called a carrier for $\bar{v}$ in $L(U, \Gamma)$.

\section{The Interval Owen Value for Fuzzy Games with a Coalition Structure and Interval Payoffs}

For a given $\bar{v} \in I G(U, \Gamma)$. According to the work of Owen [15], we define the function $\varphi: I G(U, \Gamma) \rightarrow \overline{\mathbb{R}}_{+}^{\mid \text {Supp } U \mid}$ as follows:

$$
\begin{aligned}
\varphi_{i}(U, \bar{v}, \Gamma)= & \sum_{R_{0} \subseteq M_{0} \backslash k} \sum_{S \subseteq B_{k}, i \in \operatorname{Supp} S} \alpha_{m}^{r} \beta_{B_{k}}^{S}\left(\bar{v}(S \vee Q)-{ }_{H}\right. \\
& \bar{v}((S \backslash U(i)) \vee Q)) \quad \forall i \in \operatorname{Supp} U,
\end{aligned}
$$

where $\beta_{B_{k}}^{S}=(|\operatorname{Supp} S|-1) !\left(\left|\operatorname{Supp}_{k}\right|-|\operatorname{Supp} S|\right) ! /\left|\operatorname{Supp}_{k}\right|$ ! and $\alpha_{m}^{r}=r !(m-r-1) ! / m !, m$ and $r$ respectively denote the cardinalities of $M_{0}$ and $R_{0}, Q=\vee_{l \in R_{0}} B_{l}$.

If every player's participation level is 1 , and the coalition values are real numbers, then Eq.(2) degenerates to be Eq.(1). Furthermore, if there is only one coalition in $\Gamma$, and every player's participation level is 1, then Eq.(2) degenerates to be the Shapley value given by Alparslan Gök et al.[12].

Example 3.1 Let $N=\{1,2,3,4\}, U=\{U(i)\}_{i \in N}$ with $U(i)=0.3(i \in N)$, and $\Gamma=\left\{B_{1}, B_{2}\right\}$, where $B_{1}=\{U(1)$, $U(2)\}$ and $B_{2}=\{U(3), U(4)\}$. The fuzzy coalition values are given by

$$
\begin{aligned}
& \bar{v}(U(i))=[2,4], \bar{v}(U(1), U(2))=\bar{v}(U(3), U(4))=[8,9], \\
& \bar{v}(U(i), U(j), U(k))=[15,18](i, j, k \in\{1,2,3,4\}), \\
& \bar{v}(U)=[25,30] .
\end{aligned}
$$

Since there does not exist interval number $\bar{C}$ such that $\bar{v}(U(1))+\bar{c}=\bar{v}(U(1), U(2))$, we can not use the Hukuhara difference in this game. If we adopt the extension Hukuhara difference between interval numbers, by Eq.(2) we have $\varphi_{i}(U, \bar{v}, \Gamma)=[6.25,7.25]$ $(i \in N)$. Since this is a symmetry game and every player can get more payoffs than that by himself, we know this cooperative game can be formed. Furthermore, it is not difficult to get the vector $\left(\varphi_{i}(U, \bar{v}, \Gamma)\right)_{i \in N}$ is an imputation, and an element in the core of this game.

Although the extension Hukuhara difference between interval numbers can be used to situations than the Hukuhara difference, there exist games with interval numbers which can not use the extension Hukuhara difference.
Example 3.2 In Example 3.1, if we take the values of the fuzzy coalitions as listed in table 1 .

Table 1 (The interval values of the fuzzy coalitions)

\begin{tabular}{cccc}
\hline$S$ & $\bar{v}(S)$ & $S$ & $\bar{v}(S)$ \\
\hline$\{U(1)\}$ & {$[2,10]$} & $\{U(1), U(2), U(3)\}$ & {$[28,30]$} \\
$\{U(2)\}$ & {$[4,6]$} & $\{U(1), U(3), U(4)\}$ & {$[28,30]$} \\
$\{U(3)\}$ & {$[2,10]$} & $\{U(1), U(2), U(4)\}$ & {$[24,26]$} \\
$\{U(4)\}$ & {$[4,6]$} & $\{U(2), U(3), U(4)\}$ & {$[24,26]$} \\
$\{U(1), U(2)\}$ & {$[16,18]$} & $U$ & {$[38,40]$} \\
$\{U(3), U(4)\}$ & {$[16,18]$} & & \\
\hline
\end{tabular}

From Eq.(2), we have

$$
\varphi_{2}(U, \bar{v}, \Gamma)=\varphi_{4}(U, \bar{v}, \Gamma)=[9,8]
$$

and

$$
\varphi_{1}(U, \bar{v}, \Gamma)=\varphi_{3}(U, \bar{v}, \Gamma)=[10,12] .
$$

Since the payoffs of the players 2 and 4 are "imaginary" interval numbers, which makes no sense.

In order to avoid the above situation, in this paper, for any $\bar{v} \in I G(U, \Gamma)$ we always mean every player's payoff obtained by Eq.(2) is an interval number.

Definition 3.1 Let $\bar{v} \in I G(U, \Gamma)$, a function $f: I G(U, \Gamma)$ $\rightarrow \overline{\mathbb{R}}_{+}^{\mid \operatorname{SuppU|}}$ is said to be an interval Owen value for $\bar{v}$, if it satisfies the following axioms:

Axiom 1: If $T$ is a carrier in $U$ for $\bar{v} \in \operatorname{IG}(U, \Gamma)$, then $\bar{v}(T)=\sum_{i \in \operatorname{Supp} T} f_{i}(U, \bar{v}, \Gamma) ;$

Axiom 2: Let $B_{k} \in \Gamma$ and $i, j \in \operatorname{Supp} B_{k}$, if we have $v(T \vee U(i))=v(T \vee U(j))$ for any $T \in L(U, \Gamma)$ with $i, j \notin$ $\operatorname{Supp} T$, then $f_{i}(U, v, \Gamma)=f_{j}(U, v, \Gamma)$.

Axiom 3: Let $k, s \in M_{0}=\{1,2, \ldots, m\}$, if we have $\bar{v}^{B}\left(R_{0} \cup k\right)=\bar{v}^{B}\left(R_{0} \cup k\right)$ for any $R_{0} \subseteq M_{0} \backslash\{k, s\}$, then $\sum_{i \in \operatorname{Supp} B_{k}} f_{i}(U, \bar{v}, \Gamma)=\sum_{j \in \operatorname{Supp} B_{s}} f_{j}(U, \bar{v}, \Gamma)$;

Axiom 4: Let $\bar{v}, \bar{w} \in I G(U, \Gamma)$ and all $\alpha, \beta \in \mathbb{R}_{+}$, we have $f(U, \alpha \bar{v}+\beta \bar{w}, \Gamma)=\alpha f(U, \bar{v}, \Gamma)+\beta f(U, \bar{w}, \Gamma)$.

Theorem 3.1 Let $\bar{v} \in \operatorname{IG}(U, \Gamma)$, the function $\varphi: I G(U, \Gamma) \rightarrow \overline{\mathbb{R}}_{+}^{|\operatorname{Supp} U|}$, defined by Eq.(2), is the unique interval Owen value for $\bar{v}$ in $L(U, \Gamma)$. 
Proof. From the properties of the extension Hukuhara difference between interval numbers, we have

$$
\bar{a}-{ }_{H} \bar{b}+\bar{b}=\bar{a}
$$

and

$$
(\lambda-\beta) \bar{a}=\lambda \bar{a}-{ }_{H} \beta \bar{a}
$$

for all $\bar{a}, \bar{b} \in \overline{\mathbb{R}}_{+}$and all $\lambda, \beta \in \mathbb{R}_{+}$.

According to the proof of the Owen value in crisp case, we can easily get the existence. In the following, we shall show the uniqueness.

For any $\bar{v} \in I G(U, \Gamma)$, we first show $\bar{v}$ can be expressed by

$$
\bar{v}=\sum_{\varnothing \neq T \in L(U, \Gamma)} c_{T} u_{T},
$$

where $\quad c_{T}=\sum_{P_{0} \subseteq R_{0}}(-1)_{H}^{r-p}\left(\sum_{A \subseteq S}(-1)_{H}^{\mid \text {SuppS }|-| \text { SuppA } \mid} \bar{v}(S \vee Q)\right)$, $T=S \vee_{l \in R_{0}} B_{l}$ such that $S \subseteq B_{k}$ for same $k \in M_{0}$, $R_{0} \subseteq M_{0} \backslash\{k\}$ and $u_{T}(W)=\left\{\begin{array}{cc}1 & T \subseteq W \\ 0 & \text { otherwise }\end{array}\right.$.

For any $C \in L(U, \Gamma)$, without loss of generality, suppose $C=D \vee_{l \in E_{0}} B_{l}$, where $D \subseteq B_{k}$ and $E_{0} \subseteq M_{0}$.

$$
\begin{aligned}
& \left(\sum_{\varnothing \neq T \in L(U, \Gamma)} c_{T} u_{T}\right)(C) \\
& =\sum_{\varnothing \neq T \in L(U, \Gamma)} c_{T} u_{T}(C) \\
& =\sum_{\varnothing \neq T \in L(U, \Gamma), T \subseteq C} C_{T} \\
& =\sum_{\varnothing \neq T \in L(U, \Gamma), T \subseteq C} \sum_{P_{0} \subseteq R_{0}}(-1)_{H}^{r-p}\left(\sum_{A \subseteq S}(-1)_{H}^{\mid \text {SuppS }|-| \operatorname{Supp} A \mid} \bar{v}(S \vee Q)\right) \\
& =\sum_{R_{0} \subseteq E_{0}} \sum_{P_{0} \subseteq R_{0}}(-1)_{H}^{r-p}\left(\sum_{S \subseteq D} \sum_{A \subseteq S}(-1)_{H}^{|S u p p S|-\mid \text { SuppA } \mid} \bar{V}(S \vee Q)\right) \\
& =\sum_{R_{0} \subseteq E_{0}} \sum_{P_{0} \subseteq R_{0}}(-1)_{H}^{r-p}\left(\sum_{S \subseteq D} \sum_{t=|\mathrm{Supp} S|}^{\mid \text {Supp } \mid}(-1)_{H}^{t-\mid \text { Supp } \mid}\right. \text {. }
\end{aligned}
$$

Since $\sum_{t=\mid \text { Supp } S \mid}^{\mid \text {Supp } \mid}(-1)_{H}^{t-|\operatorname{Supp} S|}\left(\begin{array}{c}|\operatorname{Supp} D|-|\operatorname{Supp} S| \\ t-|\operatorname{Supp} S|\end{array}\right)=0$ for any $S \subset D$, we get

$$
\begin{aligned}
& \left(\sum_{\varnothing \neq T \in L(U, \Gamma)} c_{T} u_{T}\right)(C) \\
& =\sum_{R_{0} \subseteq E_{0}} \sum_{P_{0} \subseteq R_{0}}(-1)_{H}^{r-p} \bar{v}(D \vee Q) \\
& =\sum_{R_{0} \subseteq E_{0}} \sum_{t=p}^{r}(-1)_{H}^{t-p}\left(\begin{array}{l}
r-p \\
t-p
\end{array}\right) \bar{v}(D \vee Q),
\end{aligned}
$$

where $r$ and $p$ denote the cardinalities of $R_{0}$ and $P_{0}$, respectively.

Since $\sum_{t=p}^{r}(-1)_{H}^{t-p}\left(\begin{array}{l}r-p \\ t-p\end{array}\right)=0$ for any $R_{0} \subset E_{0}$, we get

$$
\left(\sum_{\varnothing \neq T \in L(U, \Gamma)} c_{T} u_{T}\right)(C)=\bar{v}(C) .
$$

Thus, Eq.(3) holds.

From Axiom 4, we only need to prove the uniqueness of Eq.(2) on $u_{T}$ for any $T \in L(U, \Gamma)$.

Let

$$
M_{0}^{\prime}=\left\{j \in M_{0}: \operatorname{Supp} B_{j} \cap \operatorname{Supp} T \neq \varnothing\right\}
$$

and

$$
\operatorname{Supp} B_{j}^{\prime}=\operatorname{Supp} B_{j} \cap \operatorname{Supp} T \text {. }
$$

Define the unanimity game on $\left(M, u_{T}^{B}\right)$ as follows:

$$
u_{T}^{B}\left(R_{0}\right)=\left\{\begin{array}{ll}
1 & M_{0}^{\prime} \subseteq R_{0} \\
0 & M_{0}^{\prime} \not \subset R_{0}
\end{array},\right.
$$

where $R_{0} \subseteq M_{0}$.

Let $f$ be a solution on $\left(U, u_{T}, \Gamma\right)$ that satisfies the above axioms. From Axiom 1, we have $f_{i}\left(U, u_{T}, \Gamma\right)=0$ for any $i \notin \operatorname{Supp} T$. Furthermore, from Axiomsland 3, we obtain

$$
\sum_{i \in S u p p B_{k}} f_{i}\left(U, u_{T}, \Gamma\right)=\left\{\begin{array}{cc}
0 & k \notin M_{0}^{\prime} \\
\frac{c_{T}}{\left|M_{0}^{\prime}\right|} & k \in M_{0}^{\prime}
\end{array},\right.
$$

where $\left|M_{0}^{\prime}\right|$ denotes the cardinality of $M_{0}^{\prime}$.

By Axiom 2, we obtain

$$
f_{i}\left(U, u_{T}, \Gamma\right)=\left\{\begin{array}{cc}
0 & i \notin \operatorname{Supp} T \\
\frac{1}{\left|M_{0}^{\prime}\right|\left|\operatorname{Supp} B_{k}^{\prime}\right|} & i \in \operatorname{Supp}_{k}^{\prime}
\end{array} .\right.
$$

On the other hand, by Eq.(2) we have $\varphi_{i}\left(U, u_{T}, \Gamma\right)=0$ for any $i \notin \operatorname{Supp} T$. If $i \in \operatorname{Supp} T$, then there exists $k \in M_{0}$ such that $i \in \operatorname{Supp} B_{k}^{\prime}=\operatorname{Supp} B_{k} \cap \operatorname{Supp} T$, and

$$
u_{T}(Q \vee S \vee U(i))-u_{T}(Q \vee S)=1
$$

if and only if

$$
Q=\vee_{r \in R_{0}} B_{r},
$$

where $M_{0}^{\prime} \backslash k \subseteq R_{0} \subseteq M_{0} \backslash k$ and $\operatorname{Supp} B_{k}^{\prime} \backslash i \subseteq \operatorname{Supp} S \subseteq$ $\operatorname{Supp} B_{k} \backslash i$.

If $i \in \operatorname{Supp} B_{k}^{\prime}$, we get

$$
\begin{aligned}
& \varphi_{i}\left(U, u_{T}, \Gamma\right)=\sum_{M_{0}^{\prime} \backslash k \subseteq R_{0} \subseteq M_{0} \backslash k} \sum_{\substack{\operatorname{Supp} B_{k} \backslash i \subset \\
\text { Supp } D \subseteq S \text { Supp } B_{k} \backslash i}} \alpha_{m}^{r} \beta_{B_{k}}^{S} \\
& =\frac{1}{\left|M_{0}^{\prime} \| \operatorname{Supp} B_{k}^{\prime}\right|} \text {. }
\end{aligned}
$$

Namely, $\varphi$ and $f$ coincide on $u_{T}$.

Definition 3.2 Let $\bar{v} \in I G(U, \Gamma), \bar{v}$ is said to be convex if 
Fanyong Meng, et al

$$
\bar{v}(S \vee T)+\bar{v}(S \wedge T) \geq \bar{v}(T)+\bar{v}(S)
$$

for all $S, T \in L(U, \Gamma)$.

Definition 3.3 Let $\bar{v} \in I G(U, \Gamma)$, the vector $\bar{x}=\left(\bar{x}_{i}\right)_{i \in \text { Supp } U}$ is said to be an IPMAF for $\bar{v}$ in $L(U, \Gamma)$, if
1) $\sum_{i \in \operatorname{Supp} S} \bar{x}_{i}(S)=\bar{v}(S) \quad \forall S \in L(U, \Gamma)$,
2) $\bar{x}_{i}(S) \leq \bar{x}_{i}(T) \quad \forall i \in \operatorname{Supp} S^{\prime} \quad \forall S, T \in L(U, \Gamma)$
s.t. $S \subseteq T$,

where $S^{\prime} \subseteq B_{k}\left(k \in M_{0}\right), S=S^{\prime} \vee_{r \in R_{0}} B_{r}$ and $R_{0} \subseteq M_{0} \backslash\{k\}$.

Theorem 3.2 Let $\bar{v} \in \operatorname{IG}(U, \Gamma)$, if $\bar{v}$ is convex, then $\left(\varphi_{i}(U, \bar{v}, \Gamma)\right)_{i \in \operatorname{Supp} U}$ is an IPMAF for $\bar{v}$ in $L(U, \Gamma)$.

Proof. From Theorem 3.1, we only need to show the second condition in Definition 3.3. For all $S, T \in L(U, \Gamma)$, without loss of generality, suppose $S=S^{\prime} \vee_{r \in R_{0}^{S}} B_{r}$ and $T=T^{\prime} \vee_{r \in R_{0}^{T}} B_{r}$, where $S^{\prime} \subseteq T^{\prime} \subseteq B_{k}$ and $R_{0}^{S} \subseteq R_{0}^{T} \subseteq M_{0} \backslash\{k\}$.

From the convexity of $\bar{v} \in I G(U, \Gamma)$, we have

$$
\bar{v}(T)-_{H} \bar{v}(T \backslash U(i)) \geq \bar{v}(S)-_{H} \bar{v}(S \backslash U(i))
$$

for any $i \in \operatorname{Supp} S^{\prime}$.

Case (1). When $R_{0}^{S}=R_{0}^{T}$ and $\left|\operatorname{Supp} S^{\prime}\right|+1=\left|\operatorname{Supp} T^{\prime}\right|$, for any $W \subseteq S^{\prime}$ we have

$$
\beta_{S^{\prime}}^{W}=\sum_{K \subseteq T^{\prime} S^{\prime}} \beta_{T^{\prime}}^{W \vee K}
$$

where

$$
\beta_{S^{\prime}}^{W}=\frac{(|\operatorname{Supp} W|-1) !\left(\left|\operatorname{Supp} S^{\prime}\right|-|\operatorname{Supp} W|\right) !}{\left|\operatorname{Supp} S^{\prime}\right| !}
$$

and

$$
\beta_{T^{\prime}}^{W \vee K}=\frac{(|\operatorname{Supp}(W \vee K)|-1) !\left(\left|\operatorname{Supp} T^{\prime}\right|-|\operatorname{Supp}(W \vee K)|\right) !}{\left|\operatorname{Supp} T^{\prime}\right| !} .
$$

From Eq.(2), we get

$\varphi_{i}\left(S, \bar{v}, \Gamma_{S}\right)$

$=\sum_{H_{0} \subseteq R_{0}^{S}} \sum_{W \subseteq S^{\prime}, i \in \operatorname{Supp} W} \alpha_{R_{0}^{s}}^{H_{0}} \beta_{S^{\prime}}^{W}\left(\bar{v}(W \vee Q)-{ }_{H}\right.$

$\bar{v}((W \backslash U(i)) \vee Q))$

$\leq \sum_{H_{0} \subseteq R_{0}^{S}} \sum_{W \subseteq S^{\prime}, i \in \mathrm{Supp} W} \alpha_{R_{0}^{S}}^{H_{0}} \beta_{S^{\prime}}^{W}\left(\bar{v}(W \vee K \vee Q)-_{H}\right.$

$\bar{v}((W \backslash U(i)) \vee K \vee Q))$

$=\sum_{H_{0} \subseteq R_{0}^{S}} \sum_{W \subseteq S^{\prime}, i \in \operatorname{Supp} W} \alpha_{R_{0}^{S}}^{H_{0}} \sum_{K \subseteq T^{\prime} S^{\prime}} \beta_{T^{\prime}}^{W \vee K}\left(\bar{v}(W \vee K \vee Q)-{ }_{H}\right.$

$\bar{v}((W \backslash U(i)) \vee K \vee Q))$

$$
\begin{aligned}
= & \sum_{H_{0} \subseteq R_{0}^{S}} \alpha_{R_{0}^{S}}^{H_{0}} \sum_{W \subseteq S, i \in \operatorname{Supp} W} \sum_{K \subseteq T^{\prime} S^{\prime}} \beta_{T^{\prime}}^{W \vee K}\left(\bar{v}(W \vee K \vee Q)-{ }_{H}\right. \\
& \bar{v}((W \backslash U(i)) \vee K \vee Q)) \\
= & \sum_{H_{0} \subseteq R_{0}^{T}} \alpha_{R_{0}^{T}}^{H_{0}} \sum_{W \subseteq T^{\prime}, i \in \operatorname{Supp} W} \beta_{T^{\prime}}^{W}\left(\bar{v}(W \vee Q)-_{H}\right. \\
& \bar{v}((W \backslash U(i)) \vee Q)) \\
= & \varphi_{i}\left(T, \bar{v}, \Gamma_{T}\right),
\end{aligned}
$$

where $\alpha_{R_{0}^{T}}^{H_{0}}=\alpha_{R_{0}^{S}}^{H_{0}}=h !\left(r^{S}-h\right) ! /\left(r^{S}+1\right) !, Q=\vee_{l \in H_{0}} B, r^{S}$ and $h$ respectively denote the cardinalities of $R_{0}^{S}$ and $H_{0}$, $\Gamma_{S}$ and $\Gamma_{T}$ respectively denote the restriction of $\Gamma$ on $S$ and $T$.

From recursion method, we have

$$
\varphi_{i}\left(S, \bar{v}, \Gamma_{S}\right) \leq \varphi_{i}\left(T, \bar{v}, \Gamma_{T}\right)
$$

for any $S^{\prime} \subseteq T^{\prime} \subseteq B_{k}$ and $R_{0}^{S}=R_{0}^{T}$.

Case (2). If $\left|\operatorname{Supp} S^{\prime}\right|=\left|\operatorname{Supp} T^{\prime}\right|$ and $r^{S}+1=r^{T}$, where $r^{T}$ denotes the cardinality of $R_{0}^{T}$.

For any $H_{0} \subseteq R_{0}^{S}$, we obtain

$$
\alpha_{R_{0}^{S}}^{H_{0}}=\sum_{P_{0} \subseteq R_{0}^{T} \backslash R_{0}^{S}} \alpha_{R_{0}^{T}}^{H_{0} \cup P_{0}},
$$

where $\alpha_{R_{0}^{T}}^{H_{0} \cup P_{0}}=(h+p) !\left(r^{T}-h-p\right) ! /\left(r^{T}+1\right)$ !. p denotes the cardinality of $P_{0}$.

From Eq.(2), we have $\varphi_{i}\left(S, \bar{v}, \Gamma_{S}\right)$

$=\sum_{H_{0} \subseteq R_{0}^{s}} \sum_{W \subseteq S^{\prime}, i \in \text { Supp } W} \alpha_{R_{0}^{s}}^{H_{0}} \beta_{S^{\prime}}^{W}\left(\bar{v}(W \vee Q)-{ }_{H} \bar{v}((W \backslash U(i)) \vee Q)\right)$

$\leq \sum_{H_{0} \subseteq R_{0}^{S}} \sum_{W \subseteq S^{\prime}, i \in \text { Supp } W} \alpha_{R_{0}^{s}}^{H_{0}} \beta_{S^{\prime}}^{W}\left(\bar{v}\left(W \vee Q \vee Q^{\prime}\right)-{ }_{H}\right.$

$\left.\bar{v}\left((W \backslash U(i)) \vee Q \vee Q^{\prime}\right)\right)$

$=\sum_{H_{0} \subseteq R_{0}^{S}} \sum_{\substack{W \subseteq S^{\prime}, i \in \operatorname{Supp} W}} \sum_{P_{0} \subseteq R_{0}^{T} \backslash R_{0}^{S}} \alpha_{R_{0}^{T}}^{H_{0} \cup P_{0}} \beta_{S^{\prime}}^{W}\left(\bar{v}\left(W \vee Q \vee Q^{\prime}\right)-_{H}\right.$

$\left.\bar{v}\left((W \backslash U(i)) \vee Q \vee Q^{\prime}\right)\right)$

$=\sum_{H_{0} \subseteq R_{0}^{S}} \sum_{P_{0} \subseteq R_{0}^{T} \backslash R_{0}^{S}} \alpha_{R_{0}^{T}}^{H_{0} \cup P_{0}} \sum_{\substack{W \subseteq T^{\prime} \\ i \in \operatorname{Supp} W}} \beta_{T^{\prime}}^{W}\left(\bar{v}\left(W \vee Q \vee Q^{\prime}\right)-{ }_{H}\right.$

$\left.\bar{v}\left((W \backslash U(i)) \vee Q \vee Q^{\prime}\right)\right)$

$=\sum_{H_{0} \subseteq R_{0}^{T}} \alpha_{R_{0}^{T}}^{E_{0}} \sum_{W \subseteq T^{\prime}, i \in \operatorname{Supp} W} \beta_{T^{\prime}}^{W}\left(\bar{v}(D \vee Q)-{ }_{H} \bar{v}((D \backslash U(i)) \vee Q)\right)$

$=\varphi_{i}\left(T, \bar{v}, \Gamma_{T}\right)$,

where $Q^{\prime}=\vee_{l \in P_{0}} B_{l}$.

From recursion method, we have

$$
\varphi_{i}\left(S, \bar{v}, \Gamma_{S}\right) \leq \varphi_{i}\left(T, \bar{v}, \Gamma_{T}\right)
$$

for any $S^{\prime}=T^{\prime} \subseteq B_{k}$ and $R_{0}^{S} \subseteq R_{0}^{T}$.

By cases (1) and (2), the conclusion is obtained. 
Definition 3.4 Let $\bar{v} \in I G(U, \Gamma)$, the core $I C(U, \bar{v}, \Gamma)$ of $\bar{v}$ is defined by

$$
\begin{aligned}
I C(U, \bar{v}, \Gamma)=\left\{\bar{x}=\left\{\bar{x}_{i}\right\}_{i \in \operatorname{Supp} U} \mid \sum_{i \in \operatorname{Supp} U} \bar{x}_{i}=\bar{v}(U),\right. \\
\\
\left.\sum_{i \in \operatorname{Supp} S} \bar{x}_{i} \geq \bar{v}(S), \forall S \in L(U, \Gamma)\right\} .
\end{aligned}
$$

Theorem 3.3 Let $\bar{v} \in I G(U, \Gamma)$, if $\bar{v}$ is convex, then $\left(\varphi_{i}(U, \bar{v}, \Gamma)\right)_{i \in \operatorname{Supp} U} \in I C(U, \bar{v}, \Gamma)$.

Proof. From Theorem 3.1, we get

$$
\sum_{i \in \operatorname{Supp} U} \varphi_{i}(U, \bar{v}, \Gamma)=\bar{v}(U)
$$

By the convexity of $\bar{v}$ and Theorem 3.2, we have

$$
\bar{v}(S)=\sum_{i \in \text { SuppS }} \varphi_{i}(S, \bar{v}, \Gamma) \leq \sum_{i \in \text { Supps }} \varphi_{i}(U, \bar{v}, \Gamma)
$$

for any $S \in L(U, \Gamma)$.

Namely, $\left(\varphi_{i}(U, \bar{v}, \Gamma)\right)_{i \in \operatorname{Supp} U} \in I C(U, \bar{v}, \Gamma)$.

If there is only one coalition in $\Gamma$, and the coalition values are real numbers, then IPMAF degenerates to be fuzzy population monotonic allocation function introduced by Tsurumi et al. [4].

Furthermore, if there is only one coalition in $\Gamma$, and the coalition values are real numbers, then the core $I C(U, \Gamma)$ degenerates to be the core of fuzzy games presented by $\mathrm{Yu}$ and Zhang [25].

\section{A Special Kind of Fuzzy Games with a Coalition Structure and Interval Payoffs}

In this section, we shall discuss a special kind of fuzzy games with a coalition structure and interval payoffs, which can be seen as an extension of fuzzy games presented by Tsurumi et al. [4]. Similar to Tsurumi et al. [4], we give the fuzzy coalition value as follows:

$$
\bar{v}_{C}(S)=\sum_{l=1}^{q(S)} \bar{v}_{0}\left([S]_{h_{l}}\right)\left(h_{l}-h_{l-1}\right),
$$

where $S$ is a fuzzy coalition as usual. $Q(S)=\{U(i) \mid U(i)>0$, $i \in \operatorname{Supp} S\}$ and $[S]_{h_{l}}=\left\{i \in \operatorname{Supp} S \mid U(i) \geq h_{l}\right\} \cdot q(S)$ denote $s$ the cardinality of $Q(S)$, and the elements in $Q(S)$ are written in the increasing order as $0=h_{0} \leq h_{1} \leq \ldots \leq h_{q(S)}$. $\bar{v}_{0}$ is the associated game of $\bar{v} \in I G(U, \Gamma)$ defined on $[U]_{h_{l}}$ for any $l \in\{1,2, \ldots, q(U)\}$.

From Eq.(4), we know it is equivalent to

$$
\bar{v}_{C}(S)=\sum_{l=1}^{q(S)}\left(\bar{v}_{0}\left([S]_{h_{l}}\right)-_{H} \bar{v}_{0}\left([S]_{h_{l+1}}\right)\right) h_{l} .
$$

Let $I G_{C}(U, \Gamma)$ be the set of this kind of fuzzy games on $(U, \Gamma)$ with interval payoffs.

Similar to Definition 3.1, we give the following definition for the interval Owen value in $I G_{C}(U, \Gamma)$.

Definition 4.1 Let $\bar{v}_{C} \in I G_{C}(U, \Gamma)$, a function $f: I G_{C}(U, \Gamma)$ $\rightarrow \overline{\mathbb{R}}_{+}^{|S u p p U|}$ is said to be an interval Owen value for $\bar{v}_{C}$, if it satisfies the following axioms:

Axiom 1: If $T$ is a carrier for $\bar{v}_{C}$ in $U$, then

$$
\bar{v}_{C}(T)=\sum_{i \in \operatorname{Supp} T} f_{i}\left(U, \bar{v}_{C}, \Gamma\right) ;
$$

Axiom 2: Let $B_{k} \in \Gamma$ and $i, j \in\left[B_{k}\right]_{h_{i}}$, where $l \in\{1,2, \ldots, q(U)\}$. If we have $\bar{v}_{0}\left(S_{0} \cup i\right)=\bar{v}_{0}\left(S_{0} \cup j\right)$ for any $S_{0} \in L\left([U]_{h_{1}}, \Gamma\right)$ with $i, j \notin S_{0}$, then

$$
f_{i}\left([U]_{h_{l}}, \bar{v}_{0}, \Gamma\right)=f_{j}\left([U]_{h_{l}}, \bar{v}_{0}, \Gamma\right) ;
$$

Axiom 3: Let $l \in\{1,2, \ldots, q(U)\}, M_{0}^{h}=\left\{j \in M_{0} \mid\left[B_{j}\right]_{h_{h}} \neq \varnothing\right\}$ and all $k, s \in M_{0}^{h_{y}}$ with $k \neq s$. If $\bar{v}_{0}^{[B]_{h_{l}}}\left(R_{0} \cup k\right)=$ $\bar{v}_{0}^{[B]_{h_{\eta}}}\left(R_{0} \cup s\right)$ for all $R_{0} \subseteq M_{0}^{\eta_{i}} \backslash\{k, s\}$, then

$$
\sum_{i \in\left[B_{k}\right]_{h_{h}}} f_{i}\left([U]_{h_{l}}, \bar{v}_{0}, \Gamma\right)=\sum_{j \in\left[B_{s}\right]_{h_{h}}} f_{j}\left([U]_{h_{1}}, \bar{v}_{0}, \Gamma\right)
$$

Axiom 4: Let $\bar{w}_{C}, \bar{v}_{C} \in I G_{C}(U, \Gamma)$, then

$$
f\left(U, \bar{v}_{C}+\bar{w}_{C}, \Gamma\right)=f\left(U, \bar{v}_{C}, \Gamma\right)+f\left(U, \bar{w}_{C}, \Gamma\right),
$$

where $\left([U]_{h_{1}}, \Gamma\right)$ is a coalition structure in $[U]_{h_{l}}$ w.r.t. $(U, \Gamma)$, and $L\left([U]_{h_{l}}, \Gamma\right)$ denotes the set of all feasible coalitions in $\left([U]_{h_{h}}, \Gamma\right) \cdot \bar{v}_{0}$ is the associated game of $\bar{v}_{C} \in$ $I G_{C}(U, \Gamma)$ defined in $[U]_{h_{l}}$ for any $l \in\{1,2, \ldots, q(U)\}$.

Theorem 4.1 Let $\bar{v}_{C} \in I G_{C}(U, \Gamma)$, the function $\varphi^{C}$ : $I G_{C}(U, \Gamma) \rightarrow \overline{\mathbb{R}}_{+}^{|\operatorname{supp} U|}$, defined by

$$
\varphi_{i}^{C}\left(U, \bar{v}_{C}, \Gamma\right)=\sum_{l=1}^{q(U)} \varphi_{i}\left([U]_{h_{l}}, \bar{v}_{0}, \Gamma\right)\left(h_{l}-h_{l-1}\right) \forall i \in \operatorname{Supp} U,
$$

where

$$
\begin{aligned}
& \varphi_{i}\left([U]_{h_{l}}, \bar{v}_{0}, \Gamma\right)=\sum_{R_{0} \subseteq M_{0}^{h_{i}} \backslash k} \sum_{i \in S_{0}, S_{0} \subseteq\left[B_{k}\right] l_{h}} \alpha_{m_{H}}^{r} \beta_{\left[B_{k}\right] l_{m}}^{S_{0}}\left(\bar{v}_{0}\left(S_{0} \cup Q_{0}\right)-_{H}\right. \\
& \left.\bar{v}_{0}\left(\left(S_{0} \cup Q_{0}\right) \backslash i\right)\right), \alpha_{m_{m_{l}}}^{r}=r !\left(m_{h_{l}}-r-1\right) ! / m_{h_{l}} !, \beta_{\left[B_{k}\right]_{h_{l}}}^{S_{0}}= \\
& (s-1) !\left(\left|\left[B_{k}\right]_{h_{h}}\right|-s\right) ! /\left|\left[B_{k}\right]_{h_{h}}\right| !, Q_{0}=\bigcup_{r \in R_{0}}\left[B_{r}\right]_{h_{h}} \text { and } \\
& M_{0}^{h_{l}}=\left\{j \in M_{0} \mid\left[B_{j}\right]_{h_{l}} \neq \varnothing\right\} \quad . \quad m_{h_{l}} \quad \text { and }\left|\left[B_{k}\right]_{h_{l}}\right|
\end{aligned}
$$


respectively denote the cardinalities of $M_{0}^{h_{l}}$ and $\left[B_{k}\right]_{h_{l}}$, and $\bar{v}_{0}$ is the associated game of $\bar{v}_{C}$. Then $\varphi^{C}$ is the unique interval Owen value for $\bar{v}_{C}$ in $L(U, \Gamma)$.

From Eq.(4) and Definition 2.6, we know $\left\{\left[B_{j}\right]_{h_{1}}\right\}_{j \in M_{0}}$ is a coalition structure in $[U]_{h_{h}}$, and the set of all feasible coalitions in $\left([U]_{h_{1}}, \Gamma\right)$ is different w.r.t. the different $l \in\{1,2, \ldots, q(U)\}$.

If there is only one coalition in $\Gamma$, and the coalition values are real numbers, then Eq.(5) degenerates to be the Shapley value proposed by Tsurumi et al.[4].

Proof. Axiom 1. From Theorem 4 in [4], we know $T$ is a carrier in $U$ for $\bar{v}_{C}$ if and only if $[T]_{h_{l}}$ is a carrier in $[U]_{h_{1}}$ for $\bar{v}_{0}$, where $l=1,2, \ldots, q(U)$.

For any $S_{0} \subseteq\left[B_{k}\right]_{h_{1}}$, let

$$
\bar{v}_{0}^{Q_{0}}\left(S_{0}\right)=\bar{v}_{0}\left(S_{0} \cup Q_{0}\right){ }_{H} \bar{v}_{0}\left(Q_{0}\right) .
$$

From Eq.(5), we get

$$
\begin{aligned}
& \sum_{i \in \operatorname{Supp} T} \varphi_{i}^{C}\left(U, \bar{v}_{C}, \Gamma\right) \\
& =\sum_{i \in \operatorname{Supp} U} \varphi_{i}^{C}\left(U, \bar{v}_{C}, \Gamma\right)
\end{aligned}
$$

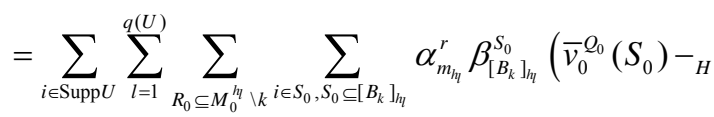

$$
\begin{aligned}
& \left.\bar{v}_{0}^{Q_{0}}\left(S_{0} \backslash i\right)\right)\left(h_{l}-h_{l-1}\right)
\end{aligned}
$$

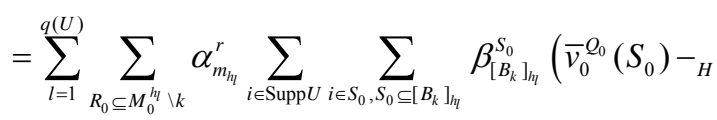

$$
\begin{aligned}
& \left.\bar{v}_{0}^{Q_{0}}\left(S_{0} \backslash i\right)\right)\left(h_{l}-h_{l-1}\right) \\
& =\sum_{l=1}^{q(U)} \sum_{R_{0} \subseteq M_{0}^{h_{\eta}} \backslash k} \alpha_{m_{m_{l}}}^{r} \bar{\nabla}_{0}^{Q_{0}}\left(\left[B_{k}\right]_{h_{l}}\right)\left(h_{l}-h_{l-1}\right) \\
& =\sum_{l=1}^{q(U)} \sum_{R_{0} \subseteq M_{0}^{\eta_{l}} \backslash k} \alpha_{m_{h_{l}}}^{r}\left(\bar{v}_{0}\left(\left[B_{k}\right]_{h_{l}} \cup Q_{0}\right)-_{H} \bar{v}_{0}\left(\left[B_{k}\right]_{h_{l}}\right)\right) \\
& \times\left(h_{l}-h_{l-1}\right) \\
& =\sum_{l=1}^{q(U)} \sum_{R_{0} \subseteq M_{0}^{h_{\eta}} \backslash k} \alpha_{m_{h_{l}}}^{r}\left(\bar{v}_{0}^{[B]_{h_{m}}}\left(R_{0} \cup k\right)-_{H} \bar{v}_{0}^{[B]_{\eta_{\eta}}}\left(R_{0}\right)\right) \\
& \times\left(h_{l}-h_{l-1}\right) \\
& =\sum_{l=1}^{q(U)} \bar{v}_{0}^{[B]_{h_{\eta}}}\left(M_{0}^{h_{l}}\right)\left(h_{l}-h_{l-1}\right) \\
& =\sum_{l=1}^{q(U)} \bar{v}_{0}\left([U]_{h_{l}}\right)\left(h_{l}-h_{l-1}\right)
\end{aligned}
$$

$=\sum_{l=1}^{q(U)} \bar{V}_{0}\left([T]_{h_{l}}\right)\left(h_{l}-h_{l-1}\right)$

$=\bar{v}_{C}(T)$,

where $\bar{v}_{0}^{[B]_{\eta_{H}}}$ is an interval quotient game on $M_{0}^{h_{l}}$ w.r.t. $\left([U]_{h_{1}}, \Gamma\right)$;

From Eq.(5), we have

$$
\begin{gathered}
\varphi_{i}\left([U]_{h_{l}}, \bar{v}_{0}, \Gamma\right)=\sum_{R_{0} \subseteq M_{0}^{\eta_{l} \backslash k}} \sum_{i \in S_{0}, S_{0} \subseteq\left[B_{k}\right]_{h_{\eta}}} \alpha_{m_{h_{l}}}^{r} \beta_{\left[B_{k}\right]_{h_{l}}}^{S_{0}} \\
\times\left(\bar{v}_{0}\left(S_{0} \cup Q_{0}\right)-_{H} \bar{v}_{0}\left(\left(S_{0} \cup Q_{0}\right) \backslash i\right)\right)\left(h_{l}-h_{l-1}\right)
\end{gathered}
$$

Thus, Axiom 2 holds;

By Eq.(5), we get

$$
\begin{aligned}
& \sum_{i \in\left[B_{k}\right]_{h_{l}}} \varphi_{i}\left([U]_{h_{l}}, \bar{v}_{0}, \Gamma\right)
\end{aligned}
$$

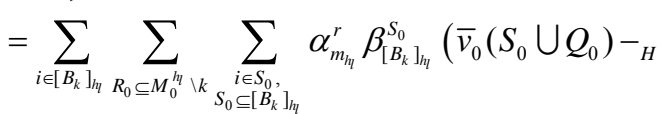

$$
\begin{aligned}
& \left.\bar{v}_{0}\left(\left(S_{0} \cup Q_{0}\right) \backslash i\right)\right)\left(h_{l}-h_{l-1}\right) \\
& =\sum_{R_{0} \subseteq M_{0}^{h_{l}} \backslash k} \alpha_{m_{\eta_{l}}}^{r}\left(\bar{v}_{0}\left(\left[B_{k}\right]_{h_{l}} \cup Q_{0}\right)-_{H} \bar{v}_{0}\left(Q_{0}\right)\right)\left(h_{l}-h_{l-1}\right) \\
& =\sum_{R_{0} \subseteq M_{0}^{\eta_{i} \backslash k}} \alpha_{m_{l_{l}}}^{r}\left(\bar{v}_{0}^{[B]_{b_{l}}}\left(R_{0} \cup k\right)-_{H} \bar{v}_{0}^{[B]_{b_{l}}}\left(R_{0}\right)\right)\left(h_{l}-h_{l-1}\right) \\
& =\sum_{R_{0} \subseteq M_{0}^{\eta_{i}} \backslash S} \alpha_{m_{m_{l}}}^{r}\left(\bar{v}_{0}^{[B]_{b_{l}}}\left(R_{0} \cup s\right)-_{H} \bar{\nu}_{0}^{[B]_{b_{l}}}\left(R_{0}\right)\right)\left(h_{l}-h_{l-1}\right) \\
& =\sum_{j \in\left[B_{s}\right]_{h_{j}}} \varphi_{j}\left([U]_{h_{l}}, \bar{v}_{0}, \Gamma\right) \text {; }
\end{aligned}
$$

Thus, Axiom 3 holds.

From Eq.(5), one can easily get Axiom 4;

(Uniqueness) From Theorem 3.1, we know $\bar{v}_{0} \in$ $I G_{0}\left([U]_{h_{h}}, \Gamma\right)$ can be uniquely expressed by

$$
\bar{v}_{0}=\sum_{\varnothing \neq T_{0} \in L\left([U]_{m_{m}}, \Gamma\right)} c_{T_{0}} u_{T_{0}}
$$

for any $l \in\{1,2, \ldots, q(U)\}$, where

$c_{T_{0}}=\sum_{P_{0} \subseteq R_{0}}(-1)_{H}^{r-p}\left(\sum_{A_{0} \subseteq S_{0}}(-1)_{H}^{s-a} \bar{v}_{0}\left(A_{0} \vee Q_{0}\right)\right)$,

$T_{0}=S_{0} \cup_{l \in R_{0}}\left[B_{l}\right]_{h_{l}}$ and $S_{0} \subseteq\left[B_{k}\right]_{h_{l}}$ for some $k \in M_{0}^{h_{l}}$,

$u_{T_{0}}\left(W_{0}\right)=\left\{\begin{array}{cc}1 & T_{0} \subseteq W_{0} \\ 0 & \text { otherwise }\end{array}\right.$ and $R_{0} \subseteq M_{0}^{h_{i}} \backslash\{k\}$.

From Eq.(4) and Axiom 4, we only need to prove the uniqueness of Eq.(5) on game $c_{S_{0}} u_{S_{0}}$ for any $S_{0} \in L\left([U]_{h_{l}}, \Gamma\right)$. Similar to Theorem 3.1, we have $\varphi_{i}\left([U]_{h_{l}}, c_{S_{0}} u_{S_{0}}, \Gamma\right)=\left\{\begin{array}{cc}0 & \text { otherwise } \\ \frac{c_{S_{0}}}{\left|M_{h_{l}}^{\prime}\right|\left|\left[B_{k}\right]_{h_{l}}^{\prime}\right|} & i \in\left[B_{k}\right]_{h_{l}}^{\prime}, k \in M_{0}^{h_{1}^{\prime}},\end{array}\right.$ 
where $M_{0}^{h_{1}{ }^{\prime}}=\left\{j \in M_{0}^{h_{1}}:\left[B_{j}\right]_{h_{1}} \cap S_{0} \neq \varnothing\right\}$ and $\left[B_{j}\right]_{h_{1}}^{\prime}=$ $\left[B_{j}\right]_{h_{l}} \cap S_{0} \cdot \square$

Theorem 4.2 Let $\bar{v}_{C} \in I G_{C}(U, \Gamma)$, the function $\phi^{C}: I G_{C}(U, \Gamma) \rightarrow \overline{\mathbb{R}}_{+}^{|S u p p U|}$, defined by

$$
\begin{gathered}
\phi_{i}^{C}\left(U, \bar{v}_{C}, \Gamma\right)=\sum_{l=1}^{q(U)}\left(\phi_{i}\left([U]_{h_{l}}, \bar{v}_{0}, \Gamma\right)-_{H} \phi_{i}\left([U]_{h_{l+1}}, \bar{v}_{0}, \Gamma\right)\right) h_{l} \\
\forall i \in \operatorname{Supp} U,
\end{gathered}
$$

where

$\phi_{i}\left([U]_{h_{l}}, \bar{v}_{0}, \Gamma\right)=\sum_{R_{0} \subseteq M_{0}^{h_{l}} \backslash k} \sum_{i \in S_{0} \subseteq\left[B_{k}\right]_{h_{l}}} \alpha_{m_{h_{l}}}^{r} \beta_{\left[B_{k}\right]_{h_{l}}}^{S_{0}}\left(\bar{v}_{0}\left(S_{0} \cup Q_{0}\right)-_{H}\right.$ $\left.\bar{v}_{0}\left(\left(S_{0} \cup Q_{0}\right) \backslash i\right)\right), \alpha_{m_{h_{l}}}^{r}$ and $\beta_{\left[B_{k}\right]_{h_{l}}}^{S_{0_{1}}}$ as given in Theorem 4.1. Then $\phi^{C}$ is equivalent to $\varphi^{C}$.

Proof. From Eq.(6), we have

$$
\begin{aligned}
& \phi_{i}^{C}\left(U, \bar{v}_{C}, \Gamma\right) \\
&=\sum_{l=1}^{q(U)}\left(\phi_{i}\left([U]_{h_{l}}, \bar{v}_{0}, \Gamma\right)-_{H} \phi_{i}\left([U]_{h_{l+1}}, \bar{v}_{0}, \Gamma\right)\right) h_{l} \\
&=\left(\phi_{i}\left([U]_{h_{1}}, \bar{v}_{0}, \Gamma\right)-_{H} \phi_{i}\left([U]_{h_{2}}, \bar{v}_{0}, \Gamma\right)\right) h_{1}+ \\
&\left(\phi_{i}\left([U]_{h_{2}}, \bar{v}_{0}, \Gamma\right)-_{H} \phi_{i}\left([U]_{h_{3}}, \bar{v}_{0}, \Gamma\right)\right) h_{2}+ \\
& \cdots+\phi_{i}\left([U]_{h_{q(U)}}, \bar{v}_{0}, \Gamma\right) h_{q(U)} \\
&=\left(\phi_{i}\left([U]_{h_{1}}, \bar{v}_{0}, \Gamma\right) h_{1}-_{H} \phi_{i}\left([U]_{h_{2}}, \bar{v}_{0}, \Gamma\right) h_{1}\right)+ \\
&\left(\phi_{i}\left([U]_{h_{2}}, \bar{v}_{0}, \Gamma\right) h_{2}-{ }_{H} \phi_{i}\left([U]_{h_{3}}, \bar{v}_{0}, \Gamma\right) h_{2}\right)+ \\
& \cdots+\phi_{i}\left([U]_{h_{q(U)}}, \bar{v}_{0}, \Gamma\right) h_{q(U)} \\
&= \phi_{i}\left([U]_{h_{1}}, \bar{v}_{0}, \Gamma\right)\left(h_{1}-h_{0}\right)+\phi_{i}\left([U]_{h_{2}}, \bar{v}_{0}, \Gamma\right)\left(h_{2}-h_{1}\right) \\
&+\cdots+\phi_{i}\left([U]_{\left.h_{q(U)}\right)}, \bar{v}_{0}, \Gamma\right)\left(h_{q(U)}-h_{q(U)-1}\right) \\
&= \sum_{l=1}^{q(U)} \phi_{i}\left([U]_{h_{l}}, \bar{v}_{0}, \Gamma\right)\left(h_{l}-h_{l-1}\right) \\
&= \varphi_{i}^{C}(U, \bar{v}, \Gamma) . \quad \square \\
& \quad \square
\end{aligned}
$$

Definition 4.2 Let $\bar{v}_{C} \in I G_{C}(U, \Gamma)$, the vector $\bar{x}=\left\{\bar{x}_{i}\right\}_{i \in \operatorname{Supp} U}$ is said to be an IPMAF for $\bar{v}_{C}$ in $L(U, \Gamma)$, if $\bar{x}$ satisfies

1) $\sum_{i \in \text { SuppS }} \bar{x}_{i}(S)=\sum_{l=1}^{q(S)} \bar{v}_{0}\left([S]_{h_{l}}\right)\left(h_{l}-h_{l-1}\right) \quad \forall S \in L(U, \Gamma)$,

2) $\bar{x}_{i}(S) \leq \bar{x}_{i}(T), \quad \forall i \in \operatorname{Supp} S^{\prime} \quad \forall S, T \in L(U, \Gamma)$

s.t. $S \subseteq T$,

where $S^{\prime} \subseteq B_{k}\left(k \in M_{0}^{h_{l}}\right), S=S^{\prime} \vee_{q \in R} B_{q}$ and $R \subseteq M \backslash k$.
Theorem 4.3 Let $\bar{v}_{C} \in I G_{C}(U, \Gamma)$, if the associated game $\bar{v}_{0} \in I G_{0}\left([U]_{h_{1}}, \Gamma\right)$ of $\bar{v}_{C}$ is convex, then $\left(\varphi_{i}^{C}\left(U, \bar{v}_{C}, \Gamma\right)\right)_{i \in \operatorname{Supp} U}$ is an IPMAF for $\bar{v}_{C}$ in $L(U, \Gamma)$.

Proof. From Theorem 4.1, we know the first condition holds. In the following, we shall show the second condition. From $S \subseteq T$, we know $[S]_{h_{l}} \subseteq[T]_{h_{l}}$ for any $l \in\{1,2, \ldots, q(T)\}$.

By Theorem 3.2, we have $\varphi_{i}\left([S]_{h_{l}}, \bar{v}_{0}, \Gamma\right) \leq \varphi_{i}\left([T]_{h_{l}}, \bar{v}_{0}, \Gamma\right)$ for any $i \in\left[S^{\prime}\right]_{h_{l}}$ and any $l \in\{1,2, \ldots, q(T)\}$.

Thus,

$$
\begin{aligned}
\varphi_{i}^{C}\left(S, \bar{v}_{C}, \Gamma\right) & =\sum_{l=1}^{q(S)} \varphi_{i}\left([S]_{h_{l}}, \bar{v}_{0}, \Gamma\right)\left(h_{l}-h_{l-1}\right) \\
& =\sum_{l=1}^{q(T)} \varphi_{i}\left([S]_{h_{l}}, \bar{v}_{0}, \Gamma\right)\left(h_{l}-h_{l-1}\right) \\
& \leq \sum_{l=1}^{q(T)} \varphi_{i}\left([T]_{h_{l}}, \bar{v}_{0}, \Gamma\right)\left(h_{l}-h_{l-1}\right) \\
& =\varphi_{i}^{C}\left(T, \bar{v}_{C}, \Gamma\right)
\end{aligned}
$$

for any $i \in \operatorname{Supp} S^{\prime}$.

From Definition 3.4, we know the core $\operatorname{IC}\left(U, \bar{v}_{C}, \Gamma\right)$ of $\bar{v}_{C} \in I G_{C}(U, \Gamma)$ can be expressed by

$$
\begin{aligned}
\operatorname{IC}\left(U, \bar{v}_{C}, \Gamma\right)= & \left\{\bar{x}=\left\{\bar{x}_{i}\right\}_{i \in \operatorname{Supp} U} \mid \sum_{i \in \operatorname{Supp} U} \bar{x}_{i}=\right. \\
& \sum_{l=1}^{q(U)} \bar{v}_{0}\left([U]_{h_{l}}\right)\left(h_{l}-h_{l-1}\right), \sum_{i \in \operatorname{Supp} S} \bar{x}_{i} \geq \\
& \left.\sum_{l=1}^{q(S)} \bar{v}_{0}\left([S]_{h_{l}}\right)\left(h_{l}-h_{l-1}\right), \forall S \in L(U, \Gamma)\right\} .
\end{aligned}
$$

Theorem 4.4 Let $\bar{v}_{C} \in I G_{C}(U, \Gamma)$, if the associated game $\bar{v}_{0} \in I G_{0}\left([U]_{h_{l}}, \Gamma\right)$ of $\bar{v}_{C}$ is convex, then

$$
\left(\varphi_{i}^{C}\left(U, \bar{v}_{C}, \Gamma\right)\right)_{i \in \operatorname{Supp} U} \in I C\left(U, \bar{v}_{C}, \Gamma\right) .
$$

Proof. From Theorems 4.1 and 4.3, one can easily get the conclusion.

Theorem 4.5 Let $\bar{v}_{C} \in I G_{C}(U, \Gamma)$, if the associated game $\bar{v}_{0} \in I G_{0}\left([U]_{h_{1}}, \Gamma\right)$ of $\bar{v}_{C}$ is convex, then $\operatorname{IC}\left(U, \bar{v}_{C}, \Gamma\right) \neq \varnothing$, and can be expressed by

$$
C\left(U, \bar{v}_{C}, \Gamma\right)=\left\{\bar{x}=\left\{\bar{x}_{i}\right\}_{i \in \operatorname{Supp} U} \mid \sum_{i \in \operatorname{Supp} U} \bar{x}_{i}=\sum_{l=1}^{q(U)}\right.
$$




$$
\begin{aligned}
& \sum_{i \in[U]_{h_{h_{l}}}} \bar{y}_{i}^{[U]_{h_{l}}}\left(h_{l}-h_{l-1}\right), \forall \bar{y}=\left\{\bar{y}_{i}^{[U]_{h_{h}}}\right\}_{i \in[U]_{h_{h}}} \\
& \in C\left([U]_{h_{l}}, \bar{v}_{0}, \Gamma\right), \forall l=\{1,2, \ldots, q(U)\},
\end{aligned}
$$

where $C\left([U]_{h_{l}}, \bar{v}_{0}, \Gamma\right)$ denotes the core of $\bar{v}_{0}$ in $L\left([U]_{h_{l}}, \Gamma\right)$.

Proof. From Theorem 4.3 in [25], it is not difficult to get the conclusion.

If there is only one coalition in $\Gamma$, and the coalition values are real numbers, then Eq.(7) degenerates to be the core given by $\mathrm{Yu}$ and Zhang [25].

Example 4.1 There are 5 companies that cooperate to develop a new product. Namely, the set of players $N=\{1,2,3,4,5\}$. Since there exist many uncertainty factors in the process of development. The players only know the possible payoffs of the coalitions formed by applying all their resources as given in Table 2. In order to reduce risk, the players are not willing to put all their resources into this cooperation, but partial participation. Further- more, since their capabilities are different, not all coalitions can be formed. If the players' participation levels in fuzzy coalition $U$ are given by $U(1)=0.3$, $U(2)=0.6, U(3)=U(4)=0.8$ and $U(5)=0.9$.

All the formed coalitions have the coalition structure $\Gamma=\left\{B_{1}, B_{2}\right\}$, where $B_{1}=\{U(1), U(2)\}$ and $B_{2}=\{U(3)$, $U(4), U(5)\}$.

Then this is a fuzzy game with a coalition structure and interval payoffs. If the fuzzy coalition values and that of their associated crisp coalitions have the relationship as given in Eq.(4). Then, this is fuzzy game in $I G_{C}(U, \Gamma)$.

Table 2 (The interval values of the crisp coalitions)

\begin{tabular}{cccc}
\hline$S_{0}$ & $\bar{v}_{0}\left(S_{0}\right)$ & $S_{0}$ & $\bar{v}_{0}\left(S_{0}\right)$ \\
\hline$\{1\}$ & {$[1,2]$} & $\{1,2,4\}$ & {$[15,16]$} \\
$\{2\}$ & {$[1,3]$} & $\{1,2,5\}$ & {$[12,15]$} \\
$\{3\}$ & {$[2,3]$} & $\{2,3,4\}$ & {$[12,15]$} \\
$\{4\}$ & {$[2,3]$} & $\{2,3,5\}$ & {$[13,15]$} \\
$\{5\}$ & {$[1,4]$} & $\{2,4,5\}$ & {$[13,15]$} \\
$\{1,2\}$ & {$[7,8]$} & $\{3,4,5\}$ & {$[16,18]$} \\
$\{2,3\}$ & {$[4,6]$} & $\{1,3,4,5\}$ & {$[20,22]$} \\
$\{2,4\}$ & {$[4,6]$} & $\{2,3,4,5\}$ & {$[23,25]$} \\
$\{2,5\}$ & {$[5,7]$} & $\{1,2,3,4\}$ & {$[22,25]$} \\
$\{3,4\}$ & {$[5,8]$} & $\{1,2,3,5\}$ & {$[26,28]$} \\
$\{3,5\}$ & {$[4,7]$} & $\{1,2,4,5\}$ & {$[26,28]$} \\
$\{4,5\}$ & {$[4,7]$} & $\{1,2,3,4,5\}$ & {$[36,40]$} \\
$\{1,2,3\}$ & {$[15,16]$} & &
\end{tabular}

From Eq.(5), we have

$$
\begin{aligned}
& \varphi_{1}^{C}\left(U, \bar{v}_{C}, \Gamma\right)=[1.8,1.95], \\
& \varphi_{2}^{C}\left(U, \bar{v}_{C}, \Gamma\right)=[3.45,4.05], \\
& \varphi_{3}^{C}\left(U, \bar{v}_{C}, \Gamma\right)=\varphi_{4}^{C}\left(U, \bar{v}_{C}, \Gamma\right)=[5.305,5.625], \\
& \varphi_{5}^{C}\left(U, \bar{v}_{C}, \Gamma\right)=[5.13,6.25] .
\end{aligned}
$$

According to Table 2, we know the associated game $\bar{v}_{0}$ is convex, so $\left(\varphi_{i}^{C}\left(U, \bar{V}_{C}, \Gamma\right)\right)_{i=\{1,2, \ldots, 5\}}$ is an IPMAF for $\bar{v}_{C}$ in $L(U, \Gamma)$, and an element in the core.

Since the players 3 and 4 are symmetric in this game, they get the same interval payoffs. Furthermore, the Hukuhara difference between interval numbers can not be used in this example, since there does not exist interval number $\bar{c}$ such that $\bar{v}_{0}(5)+\bar{c}=\bar{v}_{0}(2,5)$.

\section{Conclusion}

Based on the extension Hukuhara difference between interval numbers, we have researched the interval Owen value for fuzzy games with a coalition structure and interval payoffs, which extends the researching scope of game theory. Meantime, some properties of the given interval Owen value are studied. In order to better understand this kind of fuzzy games, we further discuss a special kind of fuzzy games with a coalition structure and interval payoffs, which can be seen as an extension of fuzzy games given by Tsurumi et al. [4]. However, we only study the interval Owen value for this kind of fuzzy games, and it will be interesting to discuss other payoff indices on it

As a special kind of fuzzy numbers, the researching for fuzzy games with coalition structure and interval 
payoffs provides reference to fuzzy games with a coalition structure and fuzzy payoffs.

\section{Acknowledgment}

The authors gratefully thank the Editor-in- Chief Prof. Jie Lu, the Associate Editor Prof. Humberto Bustince and two anonymous referees for their valuable comments, which have much improved the paper.

\section{References}

1. L. S. Shapley, A value for n-person game (Princeton University Press, Princeton, NJ, 1953).

2. J. P. Aubin, Mathematical Methods of Game and Economic Theory (North-Holland, Amsterdam, 1982).

3. D. Butnariu, Stability and Shapley value for an n-persons fuzzy game, Fuzzy Sets Syst. 4(1) (1980) 63-72.

4. M. Tsurumi, T. Tanino and M. Inuiguchi, A Shapley function on a class of cooperative fuzzy games, Eur. J. Oper. Res. 129(3) (2001) 596-618.

5. S. J. Li and Q. Zhang, A simplified expression of the Shapley function for fuzzy game, Eur. J. Oper. Res. 196(1) (2009) 234-245.

6. F. Y. Meng and Q. Zhang, The Shapley function for fuzzy cooperative games with multilinear extension form, Appl. Math. Lett. 23(5) (2010) 644-650.

7. M. Mares, Fuzzy coalition structures, Fuzzy Sets Syst. 114(1) (2000) 23-33.

8. M. Mares and Vlach, Linear coalition games and their fuzzy extensions, Int. J. Uncertain. Fuzz. 9(3) (2001) 341-354.

9. S. Borkotokey, Cooperative games with fuzzy coalitions and fuzzy characteristic functions, Fuzzy Sets Syst. 159(2) (2008) 138-151.

10. X. H. Yu and Q. Zhang, An extension of cooperative fuzzy games, Fuzzy Sets Syst. 161(11) (2010) 1614-1634.

11. S. Z. Alparslan Gök, S. Miquel and S. Tijs, Cooperation under interval uncertainty, Math. Method Oper. Res. 69(1) (2009) 99-109.

12. S. Z. Alparslan Gök, R. Branzei and S. Tijs, The interval Shapley value: an axiomatization, Cent. Eur. J. Oper. Res. 18(2) (2010) 131-140.

13. R. Branzei, O. Branzei, S. Z. Alparslan Gök and S. Tijs, Cooperative interval games: a survey. Cent. Eur. J. Oper. Res. 18(3) (2010) 397-411.

14. H. T. Banks and M. Q. Jacobs, A differential calculus for multifunctions, J. Math. Anal. Appl. 29 (1970) 46-272.

15. G. Owen, Values of games with a priori unions (SpringerVerlag. Nueva York, 1977).

16. M. J. Albizuri, Axiomatizations of the Owen value without efficiency, Math. Soc. Sci. 55(1) (2008) 78-89.

17. S. Hart and M. Kurz, Endogenous formation of coalitions, Econometrica, 51(4) (1983) 1047- 1064.

18. G. Hamiache, A new axiomatization of the Owen value for games with coalition structures, Math. Soc. Sci. 37(3) (1999) 281-305.
19. A. B. Khmelnitskaya and E. B. Yanovskaya, Owen coalitional value without additivity axiom, Math. Method Oper. Res. 66(2) (2007) 255-261.

20. G. Owen, Characterization of the Banzhaf- Coleman index, SIAM J. Appl. Math. 35(2) (1978) 315-327.

21. J. M. Alonso-Meijide, F. Carreras, M. G. Fiestras-Janeiro and G. Owen, A comparative axiomatic characterization of the Banzhaf-Owen coalitional value, Decis. Support Syst. 43(3) (2007) 701-712.

22. R. Amer, F. Carreras and J. M. Gimenez, The modified Banzhaf value for games with coalition structure: an axiomatic characterization, Math. Soc. Sci. 43(1) (2002) $45-54$.

23. J. M. Alonso-Meijde and M. G. Fiestras-Janeiro, Modification of the Banzhaf value for games with a coalition structure, Ann. Oper. Res. 109(1-4) (2002) 213 227.

24. Y. Sprumont, Population monotonic allocation schemes for cooperative games with transferable utility, Games and Economic Behavior, 2(4) (1990) 378-394.

25. X. H. Yu and Q. Zhang, The fuzzy core in games with fuzzy coalitions, J. Comput. Appl. Math. 230(1) (2009) 173-186. 